\title{
DIFFÉRENCE TEMPORELLE, DIFFÉRENCE CULTURELLE ET STYLE DANS LES CARACTÈRES DE LA BRUYÈRE
}

\section{Pierre Force}

P.U.F. | Dix-septième siècle

$2013 / 1-n^{\circ} 258$
pages 35 à 44

ISSN 0012-4273

Article disponible en ligne à l'adresse:

http://www.cairn.info/revue-dix-septieme-siecle-2013-1-page-35.htm

Pour citer cet article :

Force Pierre, « Différence temporelle, différence culturelle et style dans les Caractères de La Bruyère », Dix-septième siècle, 2013/1 n²58, p. 35-44.

Distribution électronique Cairn.info pour P.U.F..

() P.U.F.. Tous droits réservés pour tous pays.

La reproduction ou représentation de cet article, notamment par photocopie, n'est autorisée que dans les limites des conditions générales d'utilisation du site ou, le cas échéant, des conditions générales de la licence souscrite par votre établissement. Toute autre reproduction ou représentation, en tout ou partie, sous quelque forme et de quelque manière que ce soit, est interdite sauf accord préalable et écrit de l'éditeur, en dehors des cas prévus par la législation en vigueur en France. II est précisé que son stockage dans une base de données est également interdit. 


\section{Différence temporelle, différence culturelle et style dans les Caractères de La Bruyère}

Le projet de La Bruyère dans les Caractères, exprimé par l'auteur dans la préface, est de " peindre les hommes en général ${ }^{1}$ ". Pour ce faire, La Bruyère imite un auteur grec, Théophraste. L'éloignement de l'Antiquité grecque n'est pas un obstacle, car « les hommes n’ont point changé selon le cœur et selon les passions; ils sont encore tels qu'ils étaient alors et qu'ils sont marqués dans Théophraste ${ }^{2}$ ». S'il en est ainsi, en quoi consiste l'originalité de l'auteur ? Elle tient à son style. " Chose assez remarquable ", proclame Faguet, La Bruyère " usa d'un style tout nouveau pour ne rien dire de très nouveau ». Comme le dit très justement Jules Brody, " cette remarque, dans toute sa médiocrité, définit en quelques mots le statut de La Bruyère devant trois siècles de critique ${ }^{3}$ ". Cette apparente tension entre un universalisme abstrait et un style qui privilégie le particulier et le concret semble être le point de départ obligé de toute réflexion sur La Bruyère. Comme le remarque Riffaterre, «à première vue les conventions ouvertement empruntées aux moralistes de l'Antiquité ne sont guère pertinentes aux situations et aux mœurs que La Bruyère prétend observer ». Certes, " les clichés et les citations tirées des classiques, de Juvénal par exemple, témoignent $[\ldots]$ de traits qu'on retrouve partout et dans tous les temps, mais ils le font au détriment de la couleur locale et de la couleur temporelle ${ }^{4}$. De même, on est tenté d'appliquer à La Bruyère la remarque d'Isaiah Berlin sur Racine, représentant d'une "vision uniforme de l'homme " selon laquelle il existe " une nature humaine constante, indépendante du temps, des modes passagères, et des opinions changeantes ${ }^{5} »$.

1. Jean de La Bruyère, "Préface ", dans les Caractères, introduction et notes d'Emmanuel Bury, Paris, Librairie générale française, 1995, p. 118.

2. "Discours sur Théophraste", ibid., p. 69.

3. Jules Brody, Du style à la pensée. Trois études sur les Caractères de La Bruyère, Lexington, French Forum, 1980, p. 56.

4. Michael Riffaterre, "L'Effet de vrai : La Bruyère à l'eau-forte ", PFSCL-Biblio 17, n 44, 1989, p. 10.

5. Cité par Larry Norman dans The Shock of the Ancient. Literature and History in Early Modern France, Chicago, University of Chicago Press, 2011, p. 29. 


\section{LE GOÛT DE L'ÉTRANGE}

Dans son ouvrage récent, Larry Norman prend le contre-pied de ces notions, et montre que dans la Querelle des Anciens et des Modernes, les partisans des anciens étaient paradoxalement plus proches de ce que nous appellerions aujourd'hui le relativisme historique ${ }^{6}$. Au contraire des Modernes qui appliquaient à tous les temps des critères esthétiques et moraux inflexibles, les anciens revenaient sans cesse sur l'infinie variabilité des coutumes à travers le temps et l'espace. Larry Norman montre qu'il y a chez La Bruyère une conscience aiguë de l'étrangeté des coutumes antiques, et que cette conscience de l'altérité du passé lui fait prôner " un pacte de respect mutuel entre les époques ${ }^{7}$ ». C'est ainsi qu'il interprète le passage du "Discours sur Théophraste » qui plaide pour une lecture indulgente de l'auteur grec :

Ayons donc pour les livres des anciens cette même indulgence que nous espérons nous-mêmes de la postérité, persuadés que les hommes n'ont point d'usages ni de coutumes qui soient de tous les siècles, qu'elles changent avec les temps, que nous sommes trop éloignés de celles qui ont passé, et trop proches de celles qui règnent encore, pour être dans la distance qu'il faut pour faire des unes et des autres un juste discernement ${ }^{8}$.

Une certaine tolérance pour les coutumes du passé est nécessaire pour compenser la préférence spontanée que nous avons pour les coutumes et les valeurs du présent. En ce sens, selon La Bruyère, la lecture des œuvres de l'Antiquité a une valeur éthique équivalente au "connais-toi toi-même " en morale. De même que l'amour-propre nous cache la plus grande partie de ce que nous sommes, la préférence pour le présent nous offre une image fausse de nos propres coutumes. L'expérience de l'altérité des coutumes antiques provoque un choc salutaire qui corrige nos préférences spontanées :

Que si quelques-uns se refroidissaient pour cet ouvrage moral par les choses qu'ils y voient, qui sont du temps auquel il a été écrit, et qui ne sont point selon leurs mœurs, que peuvent-ils faire de plus utile et de plus agréable pour eux que de se défaire de cette prévention pour leurs coutumes et leurs manières?

Cette expérience du passé a pour conséquence une mise à distance du présent, désormais privé de son caractère familier, et appréhendé dans toute son étrangeté :

L'on entendra parler d'une capitale d'un grand royaume où il n'y avait ni places publiques, ni bains, ni fontaines, ni amphithéâtres, ni galeries, ni portiques, ni promenoirs, qui était pourtant une ville merveilleuse. L'on dira que tout le cours de la vie s'y passait presque à sortir de sa maison pour aller se renfermer dans celle d'un autre ${ }^{10}$.

6. Larry Norman, op. cit.

7. Ibid., p. 20.

8. Jean de La Bruyère, «Discours sur Théophraste », op. cit., p. 68.

9. Ibid., p. 67.

10. Ibid. 
Selon Larry Norman, pour les partisans des anciens, "l'Antiquité définissait la distance $^{11}$ ". Autrement dit, c'est la distance temporelle qui permettait de penser la distance géographique et culturelle. C'est ainsi que Norman interprète le parallèle que La Bruyère propose entre l'étrangeté des mœurs des Grecs et l'étrangeté des mœurs des "Siamois, Chinois, Nègres ou Abyssins ${ }^{12}$ ". En effet, à propos des coutumes des Grecs, La Bruyère constate que « rien n'est plus opposé à nos mœurs que toutes ces choses; mais l'éloignement des temps nous les fait goûter, ainsi que la distance des lieux nous fait recevoir tout ce que les diverses relations ou les livres de voyages nous apprennent des pays lointains et des nations étrangères ${ }^{13}$ ". En fait, dans le "Discours sur Théophraste ", la comparaison fonctionne dans les deux sens. Selon La Bruyère, le goût de l'étrange et du nouveau se manifeste principalement dans la curiosité que nous avons pour les coutumes des peuples étrangers. La Bruyère propose de réorienter cette curiosité pour permettre une juste appréciation des coutumes antiques:

Moins rebutés par la barbarie des manières et des coutumes de peuples si éloignés, qu'instruits et même réjouis par leur nouveauté, il nous suffit que ceux dont il s'agit soient Siamois, Chinois, Nègres ou Abyssins ${ }^{14}$.

Les coutumes des Grecs nous rebutent. Celles des Chinois nous intéressent par leur étrangeté. Considérons les Grecs comme s'ils étaient des Chinois, et nous leur donnerons l'attention qu'ils méritent. Il y a chez La Bruyère une pédagogie de l'étrangeté, qui part du curieux et du nouveau en les valorisant et les dévalorisant à la fois, et va chercher le nouveau là où on l'attendrait le moins, dans les coutumes de l'Antiquité.

C'est dans ce contexte me semble-t-il qu'il faut placer les déclarations de La Bruyère sur l'universalité de la nature humaine. Le mouvement initial de la pensée est du côté de l'extrême diversité des manières d'être, fidèle en cela à Théophraste lui-même, qui commençait ses Caractères en se disant incapable de rendre compte de la diversité des coutumes, non seulement entre les Grecs et les barbares, mais encore parmi les Grecs eux-mêmes :

J'ai admiré souvent, et j'avoue que je ne puis encore comprendre, quelque sérieuse réflexion que je fasse, pourquoi toute la Grèce, étant placée sous un même ciel, et les Grecs nourris et élevés de la même manière, il se trouve néanmoins si peu de ressemblance dans leurs mœurs ${ }^{15}$.

Chez La Bruyère, ce constat de différence s'applique d'abord à la différence entre les mœurs des contemporains de Théophraste et les mœurs des Français du XVII ${ }^{\mathrm{e}}$ siècle. Le constat de similarité entre le passé et le présent intervient comme un retournement inattendu du mouvement initial :

11. Larry Norman, op. cit., p. 31.

12. Jean de La Bruyère, "Discours sur Théophraste ", op. cit., p. 69.

13. Ibid.

14. Ibid.

15. Les Caractères de Théophraste, traduits par La Bruyère, dans op. cit., p. 78. 
Or ceux dont Théophraste nous peint les mœurs dans ses Caractères étaient Athéniens, et nous sommes Français; et si nous joignons à la diversité des lieux et du climat le long intervalle des temps, et que nous considérions que ce livre a pu être écrit la dernière année de la $\mathrm{CXV}^{\mathrm{e}}$ olympiade, trois cent quatorze ans avant l'ère chrétienne, et qu'ainsi il $\mathrm{y}$ a deux mille ans accomplis que vivait ce peuple d'Athènes dont il fait la peinture, nous admirerons de nous y reconnaître nous-mêmes, nos amis, nos ennemis, ceux avec qui nous vivons, et que cette ressemblance avec des hommes séparés par tant de siècles soit si entière. En effet, les hommes n'ont point changé selon le cœur et selon les passions ${ }^{16}$.

L'éloignement de Théophraste est à la fois culturel et temporel. Le sentiment de familiarité que nous éprouvons en lisant Théophraste est présenté comme un paradoxe presque incompréhensible. Il est très étonnant que nous nous retrouvions dans un auteur grec de l'Antiquité. Cela incline à penser qu'il existe une nature humaine commune aux Grecs et à nous-mêmes, mais cette communauté de nature n'est pas affirmée dogmatiquement et a priori. Elle est proposée comme une explication à une énigme.

\section{DE L'ÉTRANGE AU FAMILIER}

Selon Gadamer, le lieu de l'herméneutique est un lieu intermédiaire entre l'étrangeté totale et l'absence totale d'étrangeté ${ }^{17}$. Le travail d'interprétation, abondamment théorisé dans la tradition humaniste, consiste à négocier la différence entre ces deux pôles. Ce qui est souvent perçu chez La Bruyère comme une tension irréconciliable entre le général et le particulier, le fond et la forme, prend tout son sens quand on l'exprime dans le vocabulaire herméneutique de la tradition humaniste (dont La Bruyère se réclame explicitement, mettant une citation d'Érasme en épigraphe aux Caractères). Premièrement, le concept d'application ${ }^{18}$, d'usage aujourd'hui principalement juridique, mais utilisé autrefois dans l'ensemble des domaines interprétatifs : une règle générale n'a de force que lorsqu'elle est suivie dans des situations concrètes et particulières. Le travail de l'interprète (celui du juge dans le domaine du droit) est de faire la liaison entre la généralité de la règle et le caractère nécessairement unique d'une situation concrète. C'est ainsi que La Bruyère présente l'entreprise de Théophraste. Entre Aristote qui énonçait des principes généraux sur la nature humaine, et Galien qui, comme médecin, se concentrait sur des cas particuliers, Théophraste avait choisi une voie moyenne :

Il s'en trouve d'un troisième ordre qui, persuadés que toute doctrine des mœurs doit tendre à les réformer, à discerner les bonnes d'avec les mauvaises, et à démêler dans les hommes ce qu'il y a de vain, de faible et de ridicule, d'avec ce qu'ils peuvent avoir de bon, de sain et de louable, se plaisent infiniment dans la lecture des livres qui,

16. Jean de La Bruyère, «Discours sur Théophraste", op. cit., p. 69.

17. Hans-Georg Gadamer, Vérité et méthode. Les grandes lignes d'une herméneutique philosophique, Paris, Seuil, 1996.

18. Hans-Georg Gadamer, ibid., pp. 329-333. 
supposant les principes physiques et moraux rebattus par les anciens et les modernes, se jettent d'abord dans leur application aux mœurs du temps, corrigent les hommes les uns par les autres, par ces images de choses qui leur sont si familières, et dont néanmoins ils ne s'avisaient pas de tirer leur instruction ${ }^{19}$.

L'objectif est pratique et concret (réformer les mœurs). La méthode est herméneutique : partir de principes généraux et en faire l'application (c'est le mot même qu'utilise La Bruyère) à des mours particulières, des situations humaines concrètes.

Deuxièmement, le concept d'équite ${ }^{20}$. En appliquant la loi, l'interprète doit tenir compte du fait que celle-ci n'est jamais parfaitement adaptée au cas particulier, et que la suivre trop strictement serait abusif ou injuste. Dans la tradition herméneutique de l'Antiquité et de la Renaissance, le concept d'équité est très proche de la notion rhétorique de decorum : un discours n'est efficace que si l'orateur sait l'adapter au moment et au lieu. Comme le montre Kathy Eden, l'écriture de l'histoire chez les humanistes est largement fondée sur ces notions d'équité et de decorum ${ }^{21}$. Lorsqu'il porte des jugements sur des actions du passé, l'historien doit tenir compte de la différence des coutumes, et manifester une certaine indulgence, car l'application stricte au passé des critères du présent serait injuste. Cette pensée de l'histoire est encore celle de La Bruyère. L'injonction citée plus haut : "Ayons donc pour les livres des anciens cette même indulgence que nous espérons nous-mêmes de la postéritée ${ }^{22}$ ", peut sembler vague ou moralisatrice à ceux qui ne sont pas familiers du vocabulaire humaniste. Il est clair cependant que La Bruyère recommande l'indulgence au sens technique et précis du terme. Cette indulgence est l'aquitas des rhéteurs latins et l'epieikeia des rhéteurs grecs, pour qui la justice demande de la souplesse dans l'application de la règle.

Troisièmement, le concept d'appropriation ${ }^{23}$. Comme le rappelle Kathy Eden, la tradition humaniste établissait une distinction fondamentale, remontant à Aristote, entre propriété (to oikeion) et possession (ktesis) ${ }^{24}$. Ce qui fait d'une chose ma propriété (oikeion) est le droit que j'ai de l'aliéner, autrement dit ce qui m’appartient à moi, et à aucun autre. Le propre, en ce sens, a toutes sortes de connotations affectives, liées à la maison (oikos en grec), à la famille, à l'intime, et au familier. Comprendre un texte du passé (ou le réécrire), c'est se l'approprier, autrement dit transformer un bien étranger en quelque chose qui est uniquement à soi. C'est ainsi qu'il faut comprendre les déclarations d'originalité chez La Bruyère :

Horace ou Despréaux l'a dit avant vous. - Je le crois sur votre parole; mais je l'ai dit comme mien. Ne puis-je pas penser après eux une chose vraie, et que d'autres encore penseront après moi $^{25}$ ?

19. Jean de La Bruyère, " Discours sur Théophraste ", op. cit., p. 61.

20. Hans-Georg Gadamer, op. cit., pp. 323-329.

21. Kathy Eden, "Equity and the Origins of Renaissance Historicism: the Case for Erasmus ", Yale Journal of Law and the Humanities, ${ }^{\circ}$ 5, 1993, pp. 137-145.

22. Jean de La Bruyère, "Discours sur Théophraste", op. cit., p. 68.

23. Hans-Georg Gadamer, op. cit., pp. 286-292.

24. Kathy Eden, The Renaissance Rediscovery of Intimacy, Chicago, University of Chicago Press, 2012, p. 16.

25. Jean de La Bruyère, « Des ouvrages de l'esprit », 69 (I), op. cit., p. 155. 
On interprète le plus souvent ce passage à la lumière d'une distinction abstraite entre fond et forme. Mais cette distinction est moderne, et étrangère à la culture humaniste. Il n'y a pas de fond invariant qu'on renouvellerait par des formes changeantes. Le vrai n'a de sens que lorsqu'il est appréhendé personnellement et individuellement dans un acte d'interprétation, qui est nécessairement unique. Théophraste adhère aux principes d'Aristote, mais « il se les rend propres par l'étendue qu'il leur donne, et par la satire ingénieuse qu'il en tire contre les vices des Grecs, et surtout des Athéniens ${ }^{26}$ ». De même, " je l'ai dit comme mien " est à entendre au sens fort. La simple répétition ou citation de textes anciens est l'équivalent juridique de la possession : j'ai quelque chose en main mais je n'en suis pas le légitime propriétaire. En revanche, si je sais mapproprier ces textes, ils deviennent miens et uniquement miens.

\section{UN CARACTÈRE DE DOUCEUR}

La réflexion 23 au chapitre " $\mathrm{Du}$ Cour » est une des plus souvent citées : "Être avec les gens qu'on aime, cela suffit ; rêver, leur parler, ne leur parler point, penser à eux, penser à des choses plus indifférentes, mais auprès d'eux, tout est égal ${ }^{27}$. " Riffaterre nous donne un éloquent résumé de sa réception :

C'est le premier passage que cite Roland Barthes dans sa préface de 1963 aux Caractères, le qualifiant de maxime pénétrante. Antoine Adam dans son Histoire de la littérature française au XVIT siècle y devine une confidence. Bien mieux, le baron de Charlus y trouve la seule définition convaincante du bonheur d'aimer, convaincante parce qu'elle élimine tout ce qui n'est pas la prédication exprimant le désir. Il ajoute après l'avoir citée, "l'important dans la vie n'est pas ce qu'on aime,...c'est d'aimer ", et à propos de Madame de Sévigné qui fut, dit-il, "moins à plaindre que d'autres. Elle a passé une grande partie de sa vie auprès de ce qu'elle aimait ", il rapproche de La Bruyère cette ligne de la marquise qui justement avoue parler à sa fille "de choses si légères qu'il n'y a que vous et moi qui le $[s i c]$ remarquions " - ne dirait-on pas une paraphrase de penser à des choses plus indifférentes dans la maxime de La Bruyère ? Des réactions si diverses ne laissent aucun doute sur l'impact d'un texte pourtant si dépouillé, si libre de toute rhétorique ${ }^{28}$.

Riffaterre explique l'effet de cette réflexion en remarquant son caractère abstrait. "Le vrai, dit-il, ne demande pas la représentation de particuliers, de personnages individualisés. Le vrai demande la représentation de types. En d'autres termes, la mimésis, chez La Bruyère, est la mimésis d'une abstraction ${ }^{29}$. " Le texte offre un cadre abstrait et épuré que le lecteur vient remplir : "Ce cadre, bien entendu, cette géométrie où se mêlent l'expérience personnelle, l'interprétation enrichie par la vie, moi du lecteur, c'est le type ${ }^{30}$."

26. Jean de La Bruyère, «Discours sur Théophraste ", op. cit., p. 61.

27. Jean de La Bruyère, "Du Cœur », 23 (IV), op. cit., p. 210.

28. Michael Riffaterre, loc. cit., p. 9.

29. Ibid., p. 10.

30. Ibid., p. 12. 
L'affirmation selon laquelle le texte de La Bruyère est " libre de toute rhétorique " appelle la critique. Les Caractères sont très proches d'un certain type d'éloquence antique et humaniste, que Kathy Eden qualifie d' "éloquence de l'intime ${ }^{31}$ ". Eden identifie cette éloquence au genre de la lettre familière ou intime, mais presque tout ce qu'elle dit de l'épistolaire peut s'appliquer aux Caractères. Premièrement, la lettre familière appartient au genre épidictique, c'est-à-dire que contrairement aux genres délibératif et judiciaire, elle n'est pas agonistique : il ne s'agit pas de s'opposer à un adversaire, mais simplement de décrire des qualités morales appartenant à une personne ou à un groupe, afin de les louer ou de les blâmer. D'où le caractère graphique du genre, aux deux sens du terme : tendant vers la description détaillée, et aussi vers l'écrit (contrairement aux genres délibératif et judiciaire, qui sont oraux). Enfin, la lettre intime met en avant l'ethos de son auteur, c'est-à-dire ce qu'il a d'individuel et de particulier, et elle insiste sur les particularités et les différences, de telle sorte que « la diversité des hommes engendre une diversité de styles; tel style peut plaire à tel lecteur mais non pas à un autre, ou au même lecteur certaines fois mais non pas d'autres ${ }^{32}$ ". C'est ainsi qu'il faut comprendre l'affirmation de La Bruyère selon laquelle il n’a pas voulu écrire des maximes :

Quelques-unes de ces remarques le sont [courtes et concises], quelques autres sont plus étendues : on pense les choses d'une manière différente, et on les explique par un tour aussi tout différent, par une sentence, par un raisonnement, par une métaphore ou quelque autre figure, par un parallèle, par une simple comparaison, par un fait tout entier, par un seul trait, par une description, par une peinture : de là procède la longueur ou la brièveté de mes réflexions. Ceux enfin qui font des maximes veulent être crus : je consens, au contraire, que l'on dise de moi que je n'ai pas quelquefois bien remarqué, pourvu que l'on remarque mieux ${ }^{33}$.

Il y a dans l'" éloquence de l'intime ", une liberté de ton qui tient au fait que son but est de décrire des personnes et des situations de toutes sortes de façons, avec la conscience du fait que certaines façons plairont à certains et à certains moments, et que d'autres façons plairont à d'autres en d'autres circonstances. Il s'agit là d'une entreprise mimétique, mais mimesis ne veut pas dire copie. La Bruyère propose des traits, des ébauches, dans la tradition des notationes d'Érasme, comme l'a montré Emmanuel Bury ${ }^{34}$. En ce sens, il n'y a pas "mimésis d'une abstraction» mais plus précisément c'est la mimésis même qui passe par l'abstraction. Il ne s'agit pas d'imiter un type. Le type est ce qui permet l'imitation. Le test de validité de la mimésis est la reconnaissance, c'est-à-dire le sentiment du familier : certaines descriptions sembleront vraies et familières (oui, je connais bien cela, j’ai déjà vu cela), d'autres non (je ne reconnais pas cela, cela m'est étranger) selon le moment et le lecteur.

Il y a dans ce type d'écriture un respect du lecteur, qui est traité comme un intime, et à qui on n'impose aucune manière de voir. Le mot qui revient chez La

31. Kathy Eden, op. cit.

32. Ibid., p. 56.

33. Jean de La Bruyère, "Préface ", op. cit., p. 121.

34. Emmanuel Bury, "La Bruyère et la tradition des Caractères ", Littératures classiques, n 13 (supplément), 1991, p. 12. 
Bruyère pour caractériser cette sorte d'éloquence chez Théophraste (et implicitement chez lui-même) est la douceur. Selon La Bruyère, son maître Aristote estimait en Théophraste "sur toutes choses un caractère de douceur qui régnait également dans ses mœurs et dans son style ${ }^{35} »$. Cette estime se montra dans le fait qu'Aristote donna à son disciple deux fois un nouveau nom. Une première fois, « charmé de la facilité de son esprit et de la douceur de son élocution ", il « changea son nom, qui était Tyrtame, en celui d'Euphraste, qui signifie celui qui parle bien ${ }^{36}$ ». Par la suite, le nouveau nom n'étant toujours pas à la hauteur de l'éloquence du disciple, «il l'appela Théophraste, c'est-à-dire un homme dont le langage est divin ${ }^{37}$ ». La Bruyère cite à ce propos le jugement de Cicéron, qui se réfère lui aussi à la "douceur » de Théophraste : «Qui est plus fécond et plus abondant que Platon ? plus solide et plus ferme qu'Aristote ? plus agréable et plus doux que Théophraste ? " Et dans quelques-unes de ses épîtres à Atticus, on voit que, parlant du même Théophraste, il l'appelle son ami, que la lecture de ses livres lui était familière, et qu'il en faisait ses délices ${ }^{38}$.

On ne prête souvent pas attention à ces anecdotes apparemment moralisatrices et à ces vies d'auteurs qui ornent les préfaces des auteurs classiques, mais on aurait tort de les ignorer, car elles contiennent des indications très précises, voire techniques, sur les choix rhétoriques et stylistiques de l'auteur. L'éloge de la douceur de Théophraste signale chez La Bruyère le choix d'une "éloquence de l'intime ». Il est à cet égard très symptomatique que La Bruyère cite les Lettres à Atticus, qui sont le modèle de la lettre familière, et le fait que Cicéron appelait Théophraste «son ami ». L'éloquence de l'intime est un type d'écriture qui fait du lecteur un ami, malgré la distance, qu'elle soit temporelle ou culturelle. Ou plus précisément (et c'est un paradoxe sur lequel les humanistes reviennent souvent), c'est parce qu'il y a distance qu'il est nécessaire de passer par l'écrit, et c'est l'écrit qui produit un sentiment d'intimité qui existerait à un moindre degré si l'on était en présence de la personne elle-même.

Comme le rappelle Brody, "parmi les auteurs de sa génération, La Bruyère est le seul qui se soit singularisé, ou même signalé, par son style ${ }^{39}$ ". Il eut cette distinction de son vivant même, ayant été désigné par Gilles Ménage comme "auteur d'une manière d'écrire toute nouvelle ${ }^{40} »$. La douceur qui selon La Bruyère caractérise l'écriture de Théophraste fut sans doute aussi une raison importante de la séduction que l'écriture de La Bruyère exerça sur ses contemporains. On peut saisir aussi quelque chose de cette séduction, et du sentiment de nouveauté qui l'accompagna, en lisant les réflexions de $\mathrm{La}$ Bruyère sur l'écriture épistolaire féminine. Les lettres de Balzac et de Voiture sont des modèles de goût et d'éloquence, dit La Bruyère. Cependant, elles "sont vides de sentiments qui n’ont régné que depuis leur temps, et qui doivent aux femmes leur naissance. Ce sexe va plus loin que le nôtre dans ce genre d'écrire ${ }^{41}$ ». Les épistolières ont renouvelé le genre de la lettre familière :

35. Jean de La Bruyère, "Discours sur Théophraste ", op. cit., p. 63.

36. Ibid.

37. Ibid.

38. Ibid.

39. Jules Brody, op. cit., p. 55.

40. Cité par Jules Brody, dans ibid.

41. Jean de La Bruyère, "Des ouvrages de l'esprit », 37 (IV), op. cit., p. 138. 
Elles trouvent sous leur plume des tours et des expressions qui souvent en nous ne sont l'effet que d'un long travail et d'une pénible recherche; elles sont heureuses dans le choix des termes, qu'elles placent si juste, que tout connus qu'ils sont, ils ont le charme de la nouveauté, semblent être faits seulement pour l'usage où elles les mettent ; il n'appartient qu'à elles de faire lire dans un seul mot tout un sentiment, et de rendre délicatement une pensée qui est délicate ; elles ont un enchaînement de discours inimitable, qui se suit naturellement, et qui n'est lié que par le sens ${ }^{42}$.

On comprend ici le rapprochement que le baron de Charlus faisait entre La Bruyère et Madame de Sévigné. Ces sentiments qui ne régnaient pas encore à l'époque de Balzac et de Voiture, et qui triomphent dans l'épistolaire féminin, ainsi que dans les Caractères, sont le goût de l'intime et du familier, et une attention aux détails, aux différences. Comme dans la tradition humaniste, le marqueur d'intimité est un style, ou, pour le dire autrement, le style par excellence est le style intime. Pourquoi la douceur? Parce que la douceur est une caractéristique de l'intime et du familier. Pourquoi la nouveauté ? Parce que l'éloquence de l'intime est fondée sur un rapport subtil de l'étrange et du familier. Elle part de la constatation du divers, du différent, de l'ineffable. Elle défamiliarise l'expérience du quotidien pour susciter en dernier ressort un sentiment de familiarité et de reconnaissance: oui, c'est bien cela, je ne l'avais jamais vu ainsi, mais c'est bien cela.

Le portrait de Ménalque est l'un des plus énigmatiques des Caractères. Comme le montre Riffaterre, la clé du personnage de Ménalque est son ignorance du decorum, autrement dit de la différence entre lui-même et les autres dans les rapports sociaux. Or, "Ménalque se conduit comme s'il était le contraire de l'honnête homme, alors qu'en réalité il est parfait honnête homme ${ }^{43}$ ". Ménalque est un cas limite parce qu'il ne fait pas la différence entre ce qui est à lui et ce qui est aux autres. Étant monté par distraction dans la voiture d'un autre, "Ménalque se jette hors de la portière, traverse la cour, monte l'escalier, parcourt l'antichambre, la chambre, le cabinet ; tout lui est familier, rien ne lui est nouveau; il s'assit, il se repose, il est chez soi ${ }^{44}$ ". Il s'agit bien sûr d'une démonstration par l'absurde, mais la caractéristique du parfait honnête homme est qu'il est partout chez lui. Tout ce qu'il fait, tout ce qu'il touche est oikeion, familier, voire familial. Ménalque ne fait ainsi pas de différence entre ses enfants légitimes et ses enfants illégitimes :

De même il a dessein d'élever auprès de soi un fils naturel sous le nom et le personnage d'un valet ; et quoiqu'il veuille le dérober à la connaissance de sa femme et de ses enfants, il lui échappe de l'appeler son fils dix fois le jour ${ }^{45}$.

Comme le souligne Kathy Eden, "Aristote définissait l'excellence stylistique comme oikeia - qualité dérivée de l'oikos, ou maison, et correspondant au Latin familia, laquelle comprend à la fois la relation juridique entre un propriétaire et ses

42. Ibid.

43. Michael Riffaterre, loc. cit., p. 15.

44. Jean de La Bruyère, "De l'homme », 7 (VI), op. cit., p. 395.

45. Jean de La Bruyère, "De l'homme », 7 (VI), p. 400. 
biens, et à la dimension affective d'appartenance à un lieu et à un groupe ${ }^{46}$ ". On peut lire le portrait de Ménalque, qui est chez lui où qu'il se trouve, comme un cas limite de triomphe du familier.

Le projet de " peindre les hommes en général » n'est donc pas fondé sur l'affirmation a priori d'une nature humaine invariante. Bien plutôt, il est fondé sur une conscience des pouvoirs de l'écrit, que Gadamer qualifie de "miracle ", et qui part du constat de la distance apparemment incommensurable entre le passé et le présent :

Rien n'est pure trace de l'esprit au même point que l'écriture mais rien non plus n'est aussi dépendant de l'esprit qui comprend. Dans son déchiffrement et son interprétation se produit un miracle : la transformation de quelque chose d'étranger et de mort en quelque chose d'absolument coprésent et de familier ${ }^{47}$.

Pierre ForCE

Columbia University

46. Kathy Eden, op. cit., p. 33.

47. Hans-Georg Gadamer, op. cit., p. 183 [169]. 